\title{
Manifestações oculares do paciente infectado pelo HIV atendido no Departamento de Oftalmologia da Universidade Federal de São Paulo
}

\author{
K. T. F. Matos, M. C. M. Santos, C. Muccioli
}

Trabal ho realizado no Departamento de Oftal mol ogia, Setor deÚ vea eAIDS - EPM - U niversidadeF ederal de São Paulo, São Paulo, SP.

RESUMO - OвJ etivo. Avaliar as manifestações oculares do paciente HIV positivo.

Materiais e Métodos. É um estudo retrospectivo em 1100 pacientes ambulatoriais HIV positivo em três anos com ficha padrão e exame oftalmológico completo, atendidos no Departamento de Oftalmologia da Escola Paulista de Medicina.

Resultados. Dos 1100 pacientes, 869 (79\%) eram do sexo masculino e 231 (21\%) do feminino. Os fatores de risco mais importantes foram: homossexualidade masculina em 303 (27,55\%), heterossexualidade em $272(24,73 \%)$, usuários de drogas endovenosas em $168(15,27 \%)$ e causa indeterminada ou não revelada em 246 (22,36\%). As queixas oculares mais freqüentes foram: 432 (39,27\%) pacientes com baixa da acuidade visual; 138 (12,55\%) com embaçamento visual; 64 (5,82\%) com

\section{NTR ODUÇÃO}

O envolvimento ocular que ocorre freqüentemente em pacientes infectados pelo HIV é um importante fator de morbidade ${ }^{1,2}$.

A importância da investigação ocular se dá pela alta incidência das lesões oculares associadas à gravidade de al gumas infecções na função visual ${ }^{3,4}$.

$\mathrm{O}$ aumento da média de sobrevida desses pacientes tem causado o aumento concomitante da incidência de infecções oportunistas, assim como o reconhecimento de novos agentes etiológicos.

Dentre as formas de acometimento ocular, podemos encontrar: retinopatia do HIV, infecções oportunistas secundárias propriamente ditas, manifestações neuro oftal mol ógi cas, neoplasias e lesões iatrogênicas, como nos depósitos retinianos em crianças tratadas com didanosina $(D D I)^{5}$ e nos casos de Stevens-J ohnson'.

O objetivo deste estudo éaval iar as manifestações oculares, investigar os fatores de risco, as queixas oculares, traçando, assim, um perfil oftalmológico dos pacientes infectados pelo HIV atendidos em serviço de referência num período de três anos. dor ocular, $186(16,9 \%)$ com queixas inespecíficas e $235(21,36 \%)$ pacientes não apresentavam qualquer queixa. As manifestações oculares mais freqüentes foram: retinite por CMV em 246 (22,36\%) dos pacientes; exsudatos algodonosos em 110 (10\%), blefarite em $51(4,64 \%)$ e toxoplasmose ocular em 48 (4,36\%).

Conclusão. Houve progressão da doença entre os heterossexuais e aumento da incidência no sexo feminino nesta população estudada. Os que apresentavam queixas oculares foram a maioria. A manifestação ocular mais freqüente foi a retinite por CMV em 246 (22,36\%). Encontramos 10\% de exsudatos algodonosos.

UNITERMOS: AIDS. Manifestações oculares. Uveíte. Inflamação intraocular.

\section{MATERIAIS E MÉTODOS}

No período de janeiro de 1993 a janei ro de 1996, foram atendidos 1100 pacientes HIV positivo com confirmação laboratorial pel o método de ELISA no Departamento de Ú vea e AIDS da E scola Paulista de Medicina, Universidade Federal de São Paulo, em estudo retrospectivo.

Durante a consulta foram anotados os seguintes dados: identificação, grupo de risco, doenças sistêmicas, drogas em uso, queixas oculares, exame oftalmológi co completo, diagnóstico e conduta. $\mathrm{O}$ exame oftalmológico constituiu de anamnese, determi nação da acuidade visual, ectoscopia, biomicroscopia, tonometria e oftalmoscopia binocular indireta.

\section{RESULTADOS}

Do total de pacientes examinados, 869 (79\%) eram pacientes do sexo masculino e 231 (21\%) do sexo feminino.

Os fatores de risco encontrados foram: homossexualidade masculina em 303 (27,55\%), heteros- 
Matos KTF et al.

\begin{tabular}{|lcc|}
\hline Tabela 1 - Grupo de risco & & \\
\hline & No & $\%$ \\
Homossexual & 303 & 27,55 \\
Heterossexual & 272 & 24,73 \\
Usuário de drogas EV & 168 & 15,27 \\
Transfusão de sangue & 50 & 4,54 \\
Bissexual & 37 & 3,36 \\
Perinatal & 24 & 2,18 \\
Indeterninado & 246 & 22,36 \\
Total & 1100 & 100 \\
\hline
\end{tabular}

\begin{tabular}{|lcc|}
\hline \multicolumn{2}{|l|}{ Tabela 2 - Manifestações oculares } & \\
\hline & No & $\%$ \\
Retinite por CMV & 246 & 22,36 \\
Exsudatos algodonosos & 110 & 10 \\
Blefarite & 51 & 4,64 \\
Toxoplasmose & 48 & 4,36 \\
Olho seco & 27 & 2,45 \\
Ceratite herpética & 19 & 1,73 \\
Papilite & 12 & 1,09 \\
PORN & 12 & 1,09 \\
Uveíte anterior & 9 & 0,82 \\
ARN & 7 & 0,64 \\
Lues & 5 & 0,45 \\
Glaucoma & 3 & 0,27 \\
Kaposi conjuntival & 3 & 0,27 \\
Molusco & 2 & 0,18 \\
Microsporidium & 2 & 0,18 \\
Úlcera & 2 & 0,18 \\
Tuberculose ocular & 1 & 0,09 \\
Outros & 51 & 4,64 \\
Nenhuma & 490 & 44,54 \\
\hline
\end{tabular}

sexualidade em 272 (24,73\%), usuários de drogas endovenosas em 168 (15,27\%), contaminados por transfusão sangüínea, neste caso incluindo os hemofílicos, encontramos 50 (4,54\%), bissexualidade em 37 (3,36\%), perinatal em 24 (2,18\%) e causa indeterminada ou não mencionadas pelos doentes em 246 (22,36\%).

Em relação às queixas oculares, encontramos 432 (39,27\%) pacientes com baixa da acuidade visual, excluindo-se os présbitas, 138 (12,55\%) com embaçamento visual, 64 (5,85\%) com dor ocular, 54 (4,91\%) com "floaters" ou moscas volantes, e em outros $186(16,9 \%)$ as queixas mais variadas possíveis, tais como perda do campo visual, prurido, sensação de corpo estranho e outras menos específicas, sendo que 235 (21,36\%) pacientes não apresentavam qualquer tipo de queixa.

As manifestações oculares se distribuíram da seguinte maneira: 246 (22,36\%) dos pacientes apresentaram retinite por CMV, 110 (10\%) apresentaram como única manifestação do fundo de ol ho exsudatos al godonosos, 51 (4,64\%) apresentaram bl efarites, $48(4,36 \%)$ toxopl asmose ocular em atividade (excluímos neste caso os pacientes que apresentavam lesões cicatrizadas), 27 (2,45\%) com ol ho seco, 19 (1,73\%) com ceratite herpética, 12 (1,09\%) com papilite, 12 (1,09\%) com retinite necrosante progressiva da retina externa (PORN), nove $(0,82 \%)$ com uveíte anterior, sete $(0,64 \%)$ com necrose aguda de retina (ARN), cinco $(0,45 \%)$ com lues, três $(0,27 \%)$ com glaucoma, três $(0,27 \%)$ com Sarcoma de Kaposi conjuntival, dois $(0,18 \%)$ com molusco contagiosum, dois $(0,18 \%)$ com microsporidium, dois $(0,18 \%)$ com úlcera de córnea, um $(0,09 \%)$ com tuberculose ocular e os outros 51 $(4,64 \%)$ dos paci entes apresentaram descolamento de retina em estágio terminal, phythisis, coroi dite multifocal e hemorragia retiniana, sem confirmação diagnóstica, e 490 pacientes (44,54\%) não apresentavam alteração oftal mológica.

À época da primeira avaliação oftalmológica, 407 (37\%) pacientes não apresentavam qualquer tipo de manifestação extraocular em atividade.

\section{DISCUSSÃO}

Cada vez se faz mais necessário um entendimento maior por parte dos oftalmologistas a respeito de diagnóstico, tratamento e seguimento dos paci entes infectados pel o HIV. A retinite por CMV, por si só, na ausência de outros fatores de risco, é suficiente para fazer o diagnóstico de AIDS 8.

Com a perda da efetividade do sistema imunológico, as lesões se manifestam de manei ra diversa quando comparadas com a população imunocompetente.

É interessante notar que, em relação ao trabaIho anteriormente publicado por Muccioli et al. ${ }^{7}$, notamos algumas diferenças em relação as al terações do padrão dos fatores de risco. Até Setembro de 1993, 194 pacientes atendidos eram homossexuais contra 93 heterossexuais a partir dessa data; até janei ro de 1996, 179 paci entes eram heterossexuais e 109 homossexuais, demonstrando claramente a progressão da doença entre os heterossexuais e aumento da inci dência no sexo femi nino na população examinada no ambulatório de úvea e AIDS da EPM-Unifesp que consiste em um centro de referência terciário.

N otamos também uma alta incidência de pacientes, 635 (57,72\%), com manifestações oftalmológicas na primeira consulta, o que provavelmente se justifica pelo fato de estarmos examinando uma população de pacientes encaminhados a um 


\begin{tabular}{|lcc|}
\hline \multicolumn{2}{|l|}{ Tabela 3-Queixas oculares } & \\
\hline & No & $\%$ \\
Baixa de acuidade visual & 432 & 39,27 \\
Embaçamento yisual & 138 & 12,55 \\
Dor ocular & 64 & 5,82 \\
Moscas volantes & 54 & 4,91 \\
Outros & 186 & 16,9 \\
Nenhuma & 235 & 21,36 \\
\hline
\end{tabular}

centro de referência.

A retinite por CMV no paciente com AIDS é a infecção ocular mais comum e, muitas vezes, é de início insidioso e requer uma intervenção rápida e agressiva, a fim de prevenir a perda visual. As queixas oculares se apresentavam em $79,42 \%$ dos pacientes, enquanto que $21,36 \%$ não apresentavam qualquer queixa. Quatrocentos e setenta e cinco (43\%) dos pacientes não apresentavam qualquer tipo de alteração oftalmológica, verificamos que existe um importante diferencial de $32,42 \%$ dos pacientes que apresentam queixas oculares sem alterações oftalmológicas ao exame.

As manifestações oculares foram encontradas em 635 (57,72\%) dos pacientes e a de maior incidência, 246 (22,36\%), foi a retinite por CMV, similar a trabalhos previamente publicados ${ }^{1,3,7}$.

Encontramos $10 \%$ de exsudatos algodonosos, sendo uma proporção menor que a da literatura $(25 \text { a } 40 \%)^{10}$, pois os exsudatos algodonosos são efêmeros e os dados da literatura são em função de uma avaliação longitudinal .

Encontramos lesões ativas de toxoplasmose ocuIar em 4,36\% e lesões ci catrizadas em 3,36\%, achados idênticos a relatos prévios de Muccioli et al. ${ }^{7}$ (5,16\% e 3,36\%, respectivamente).

Foi evidenciado também blefarite em $4,36 \%$ e ol ho seco em 1,72\%. Não encontramos coroidite por Pneumocisti carinii, o que pode estar relacionado ao uso profilático de sulfa em $38,18 \%$ dos pacientes.

O tratamento oftal mológico se faz necessário a fim de prevenir a perda visual, evitando a deterioração da qualidade de vida do paciente HIV positivo e, muitas vezes, servindo até como auxílio diagnóstico das manifestações sistêmicas.

\section{AGRADECIME NTOS}

Ao Dr. Bemardo Albergaria e ao Dr. Augusto Paranhos J r. pela colaboração na col eta de dados e análise crítica.

\section{SUMMARY}

Ocular manifestations of the HIV infected

\section{Patients at the Ophthalmology Department of São Paulo Federal University}

Purpose: To evaluate ocular manifestations of HIV-infected patients

Material and Methods: A restropective study of 1,100 HIV positive out patients in 3 years with a standard complete ophthal mological exam.

Results: Eigth hundred and sixty nine (79\%) of the 1,100 patients studied were male and 231 (21\%) female. The most important risk factors were: male homossexuality in 303 cases (27.55\%), heterossexuality in 272 (24.73\%), endovenous drugs use in 168 (15.27\%), and unknown cause in 246 (26.36\%).

Related to ocular complains, we found 432 (39.27\%) patients with decreased visual acuity, $138(12.55 \%)$ with visual hazy, 64 (5.82\%) with ocular pain, $186(16.9 \%)$ with inespecific complaints, and 235 (21.36\%) with no complains.

The most frequent ocular manifestations were: CMV retinitis in 246 (22.36\%), cotton wool spots in 110 (10\%), blepharitis in 51 (4.64\%), and toxoplasmosis in 48 (4.36\%).

Conclusion: The increase of disease ratio was observed among heterossexual and females in this specific population. Patients with ocular complains were $79.42 \%$. The most frequent ocular manifestations was CMV retinitis, in 246 (22.36\%). Wefound also $10 \%$ of cotton wool spots. [Rev Ass Med Bras 1999; 45(4): 323-6]

KEY-WORDS: AIDS. Ocular manifestations. Uveitis. Intraocular inflammation.

\section{REFERÊNCIAS BIBLIOGRÁFICAS}

1. Garland RF, Pickman LS, Freeman WR: Ocular Examinationand Diagnosis in Patients with the Acquired Immunodeficiency Syndrome. West J Med 1993, 158(3): 254-262.

2. Freeman WR, Helm M: Retinal and Ophthal mol ogic Manifestation of AIDS, In Ryan SP (ED). Retina, St Louis, MO, CV Mosby 1989,597-616.

3. Holland GN, Pepose J S, Pettit TH, et al . Acquired Immunedeficiency Syndrome. Ocular Manifestations. Ophthalmol 1983, 90: 859-873.

4. Bafluelos J B, Ortiz MAG, et al. Afección Ocular en los Pacientes con SIDA. Rev Cli Esp 1993, 193(7): 61-68.

5. Whitcup SM, Butler MK, Caruso R, et al. Retinal Toxicity in Human Immunodeficiency Virus-infected Children Treated with 2',3'- dideoxyiosine. Am J Ophthalmol 1992,113: 1-7.

6. Belfort J r R, De Smet M, Whitcup SM, et al. Ocular Complications of Stevens J ohnson Syndrome and Toxic Epidermal Necrolysis in Patients with AIDS. Cornea 199, 6:536-538.

7. Muciolli C, Belfort J r R, Lottemberg C, et al. Achados Oftalmológi cos em AIDS: Aval iação de 445 casos atendidos em um ano. Rev Ass Med Brasil 1994, 40 (3):155-58. 


\section{Matos KTF et al.}

8. Centers for Disease Control: Revision of the Centers for Disease Control Survellance Case Definition for Aquired Immunodeficiency Syndrome. MMWR 1987, 36(supp): IS-I6S.

9. GarianoRF, Rickman WR, Freeman WR: Ocular Examination and Diagnosis in Patients with the Acquired Immunodeficiency Syndrome. West J Med 1993, 158:254-262.

10. Pepose J S, Holland GN, Nestor MS, Cochran AJ , Foos RY: Acquired Immunedeficiency Syndrome. Pathogenic Mechanisms of Ocular Disease. Ophthalmol 1985, 92:472-484. 